\title{
Reconstruction of the Portal Vein with Expanded Polytetrafluoroethylene Jump Graft in Living Donor Liver Transplantation Recipients with Complete Portal Vein Thrombosis: A Feasible and Safe Alternative
}

\author{
Ashok Thorat ${ }^{1,5}$, Long-Bin Jeng ${ }^{1,2,5}$, Shih-Chao Hsu' ${ }^{1,2,5}$, Ping-Chun $\mathrm{Li}^{1,4,5}$, Chun-Chieh Yeh ${ }^{1,2,5}$, \\ Te-Hung Chen ${ }^{1,2,5}$, Horng-Ren Yang ${ }^{1,2,5}$, Kin-Shing Poon ${ }^{3,5}$
}

Corresponding author:

Long-Bin Jeng, MD

Organ Transplantation Center

China Medical University Hospital

2, Yuh-Der Road, Taichung, Taiwan

40447

TEL: 04-22052121Ext.1765

FAX: $04-22029083$

E-mail: otc@mail.cmuh.org.tw
${ }^{1}$ Organ transplantation centre, China Medical University Hospital, Taichung, Taiwan ${ }^{2}$ Department of Surgery, China Medical University Hospital, Taichung, Taiwan ${ }^{3}$ Department of Anaesthesiology, China Medical University Hospital, Taichung, Taiwan ${ }^{4}$ Department of cardiovascular surgery, China Medical University Hospital, Taichung, Taiwan ${ }^{5}$ China Medical University, Taichung, Taiwan

\section{ABSTRACT}

Portal vein thrombosis (PVT) increases the surgical complexity of living donor liver transplantation (LDLT) and pre-transplant complete PVT is associated with decreased 1-year patient survival. Increased experience in the field of liver transplantation $(\mathrm{LT})$ and refinements of the surgical techniques have led to successful outcomes even in recipients with complete PVT. Such techniques include thrombectomy of the native portal vein, extensive thromboendovenectomy up to the splenomesenteric confluence, venous conduit interposition and portocaval hemitransposition. In this report, we describe the outcome after LDLT using an expanded polytetrafluoroethylene (ePTFE) conduit from superior mesenteric vein (SMV) for portal inflow in patients with complete PVT.

Materials and Methods: From 10 ${ }^{\text {th }}$ September 2002 to $31^{\text {st }}$ May 2017, 900 LT surgeries were performed at China Medical University Hospital, Taiwan. The database of the 30 recipients (3.3\%) that had pre-LT PVT was retrospectively analysed. Portal vein thrombectomy was done in 27 patients, whereas a ePTFE graft was used as a jump graft from the SMV to establish portal flow in three LDLT recipients $(n=3)$.

Results: ePTFE jump graft was used to establish portal inflow in three LDLT recipients (M:F, 3:0; mean age, 53 years). The first patient in this series did not receive any anticoagulation. At $14^{\text {th }}$ month post-LDLT the ePTFE conduit was found to be thrombosed with multiple collaterals. However, the liver function tests were normal. The patient developed one episode of sepsis from which he completely recovered, and continues to have normal liver function till the latest follow up. The remaining 2 patients in this series received warfarin as anticoagulation therapy, with a dose of $3.5 \mathrm{mg}$ per day. The latest imaging studies in both the recipients revealed a patent ePTFE conduit with satisfactory portal flow and normal postoperative liver function.

Conclusions: The portal inflow can be successfully established in LDLT for recipients with pre-LT complete PVT without increasing the mortality risk. However, anticoagulation with warfarin must be instituted to avoid thrombotic occlusion of the ePTFE graft.

Key words: portal vein thrombosis, living donor liver transplantation, ePTFE graft, portal vein reconstruction 


\section{INTRODUCTION}

The reported incidence of non-malignant PVT in patients with end-stage liver disease while they are waitlisted for LT varies from $5 \%$ to $26 \%$ (1). The characteristic parenchymal changes of the cirrhotic process as well as changes in vasoreactivity result in increased intrahepatic vascular resistance and reduced portal flow. This eventually leads to altered portal hemodynamics that predisposes to PVT (2). Complete PVT was initially considered as an absolute contraindication for liver transplantation due to associated higher mortality and morbidity. After the first report by Shaw et al (3), there has been continuous refinement of the surgical techniques in order to establish portal inflow in LT in the presence of PVT. This has led to successful outcomes even in recipients with complete PVT.

PVT may be partial or complete. In partial PVT, the objective of establishing effective portal venous inflow can be achieved by attempting removal of the clot by eversion thrombectomy or thrombendvenectomy (1). After the porto-portal anastomosis is completed, the portal inflow to the graft should be measured by an immediate Doppler flowmeter analysis. The absence of inflow mandates an extra-anatomic jump graft.

The complete PVT, on the other hand, poses a technical complexity, as complete removal of the thrombus in order to establish portal flow may not be possible even if eversion thrombectomy can be extended down to the confluence of the superior mesenteric vein (SMV) and splenic vein. However, complete PVT sometimes leads to attenuated native portal vein that has reduced to a fibrotic remnant and progression of the thrombus beyond the splenomesenteric confluence. In such cases, it may be impossible to restore adequate portal inflow and an extra-anatomic portal vein anastomosis becomes necessary. In the presence of extensive PVT or when there is no suitable engorged collateral coronary vein available, a vascular conduit is used as jump graft between the distal SMV and the donor portal vein. The inter-positional graft can be the donor iliac vein, or cryopreserved vascular grafts. However, in the absence of such sources, the complexity of portal vein reconstruction increases. The synthetic vascular grafts such as ePTFE seem to be a good alternative for outflow venoplasty, when autologous or allogeneic vascular patch are not available in the context of extensive porto-mesenteric thrombosis. However, such experience remains rarely reported (4).

Use of ePTFE as a jump graft for portal inflow reconstruction in LDLT recipients with complete PVT are rarely reported and outcomes in this regard remains limited. Herein we describe our experience with using ePTFE synthetic graft for inflow reconstruction in LDLT recipients with extensive PVT.

\section{MATERIALS AND METHODS}

From $10^{\text {th }}$ September 2002 to $31^{\text {st }}$ May 2017, 900 LT surgeries were performed at China Medical University Hospital, Taiwan. The data of the 30 recipients (3.3\%) that had pre-LT PVT was retrospectively analysed. Portal vein thrombectomy was performed in 27 patients whereas ePTFE graft was used as a jump graft from SMV to establish portal flow in three LDLT recipients $(n=3)$. The demographic data, medical records and pre-transplant laboratory data of the recipients were assessed for any abnormality. The complications directly related to HA were recorded and retrospectively analysed. Triphasic computed tomography (CT) scan of the liver was done in all the patients. The presence of PVT was confirmed in the pre-transplant period and the extent of the thrombosis was assessed in order to define the surgical strategy for the portal vein reconstruction during the transplant surgery.

During the recipient explant hepatectomy, thrombendvenectomy for PVT was attempted before using ePTFE graft as a conduit. The first patient of this series received no anticoagulation except Aspirin $75 \mathrm{mg}$ once daily for two years. However, after the initial experience, we changed the protocol for PV reconstruction using ePTFE graft. Hence, warfarin was used as an anticoagulant for the last two patients. The anticoagulation treatment was aimed to keep the international normalized ratio (INR) between 2 and 2.5. All patients received standard immunosuppressive therapy, which included induction therapy with simulect, intravenous steroids (which were gradually tapered and stopped completely at two weeks post-transplantation), tacrolimus and everolimus (5).

\section{Surgical technique of using ePTFE as a supra-duodenal jump graft}

The explant surgery of the recipient followed a standard surgical technique. The portal venous flow in all the recipients of this case series was absent as detected by intraoperative Doppler ultrasound and venous collateral flow was not prominent. Mechanical thrombectomy was not possible due to complete PVT that extended down to the splenomesenteric junction. The artificial vascular graft used in each case was a 10 mm-diameter ePTFE (Gore-Tex Propaten Vascular 
Graft, Inc United States). Immediately after the explant hepatectomy was completed, superior mesenteric vein (SMV) access was secured in the infracolic compartment at the lower border of the pancreas, after opening the lesser sac. The SMV was mobilized proximally and isolated circumferentially in order to facilitate the positioning of the vascular clamp. Distal end of SMV was closed with 4-0 prolene sutures at splenomesenteric junction. After heparinization of the proximal SMV, its stump was anastomosed to the ePTFE graft in an end-to-end fashion, after placing the vascular clamp proximally (fig. $1 a, b)$ ). The ePTFE graft was brought through the mesocolon as a jump graft and placed anterior to the first part of duodenum. At this point of surgery, the liver allograft was taken out of the cold basin and cold ischemia timing was noted. The outflow reconstruction was done by side to side cavocaval anastomosis (modified piggyback technique). The portal vein was prepared for inflow reconstruction. The vascular clamp on the proximal SMV was momentarily removed to check the blood flow from the distal end of ePTFE. The distal end of ePTFE graft was then anastomosed to graft portal vein in similar end-to-end fashion using 4-0 prolene suture by standard suturing technique (fig. $1 \mathrm{c}$ ). No growth factor was given while tying the knot at completion of the suturing. The adequacy of portal vein inflow was measured using a Doppler flowmeter after completion of the vascularreconstruction and was repeated at the endof the recipient surgery before closing the abdomen. If any flow disturbances were detected, they were addressed immediately.

Right liver allografts were used for all of the recipients, and middle hepatic vein (MHV) reconstruction was done in all the three liver allografts using ePTFE vascular grafts during the backtable procedure, as described earlier (6).

\section{RESULTS}

The ePTFE jump graft was used to establish portal inflow in three LDLT recipients (M:F, 3:0; mean age, 53 years). The indications for the LDLT were cryptogenic liver cirrhosis in 2 patients and viral hepatitis B end stage liver disease in one patient. A right liver allograft was implanted in all three patients. The average warm ischemia time was 49 minutes, while cold ischemia time was 93 minutes. The anhepatic phase lasted for an average of 76 minutes for all the patients. Portal vein reconstruction time was 36 minutes. The average blood loss was $4200 \mathrm{ml}$ without any hemodynamic instability recorded during surgery. The postoperative recovery
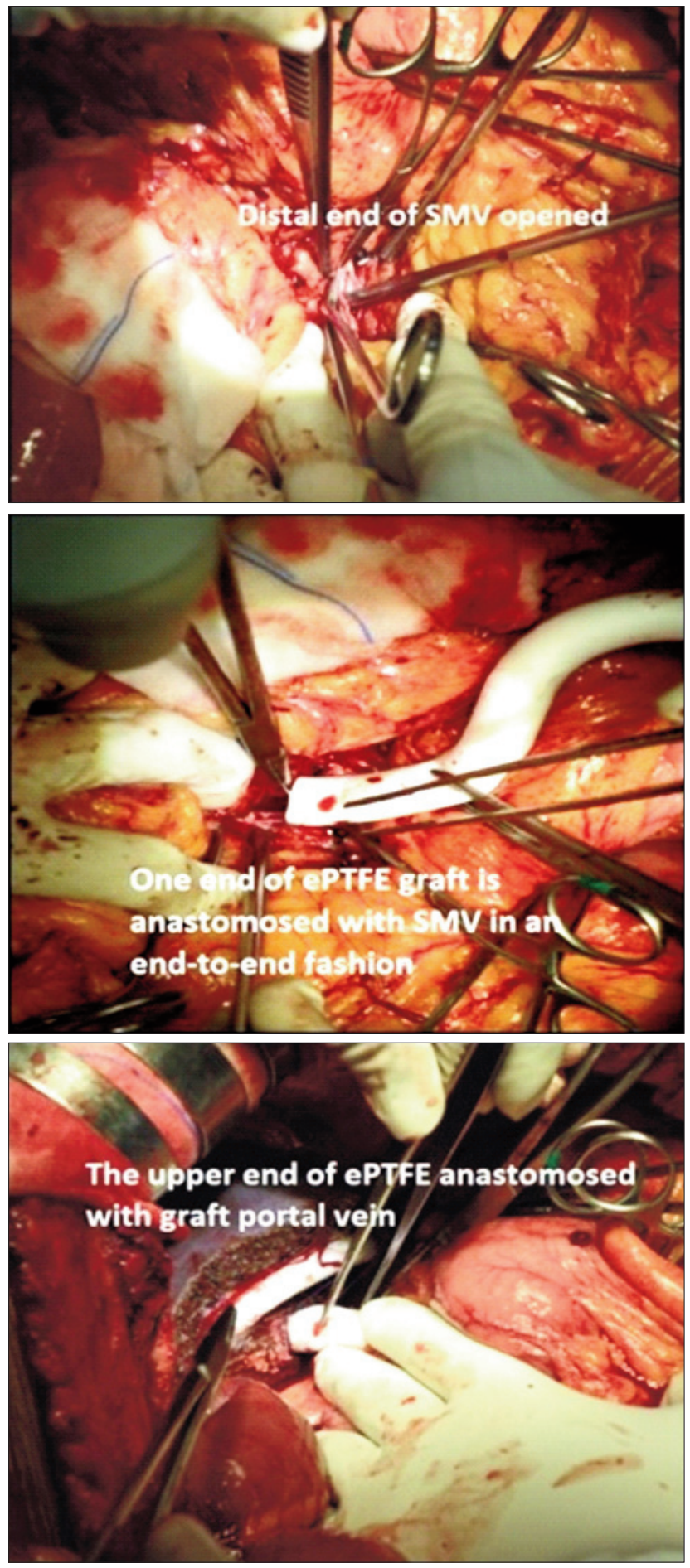

Figure 1 - (a) - The distal end of the SMV opened in lesser sac at lower border of the pancreas; (b) - one end of ePTFE graft anastomosed to SMV in end-to-end fashion; (c) - The upper end of the ePTFE anastomosed to the graft portal vein

was uneventful in all patients. Liver function tests gradually normalized in the post-transplant period. None of the patients had acute rejection and infectious complications were nil for all of the three patients. Postoperative colour Doppler study as per institutional 


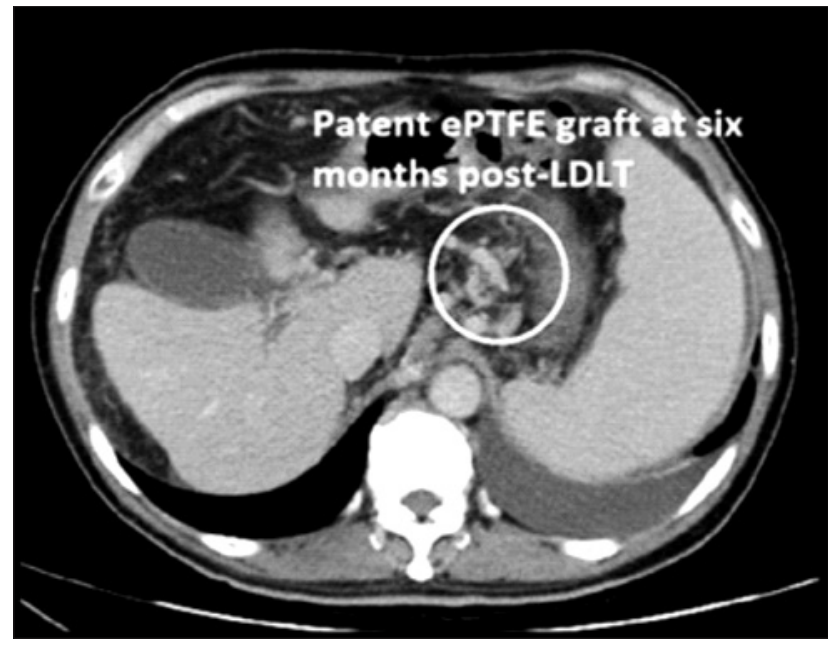

Figure 1d - Post-operative follow up CT scan shows patent graft

protocol revealed normal portal inflow and outflow. The first patient in this series did not receive any anticoagulation. At 14 months post-LDLT the ePTFE conduit was found to be thrombosed, with multiple collaterals. However, the liver function tests were normal. The patient developed one episode of sepsis from which he completely recovered, and continued to have normal liver function up to the latest follow up. The remaining two patients in this series received warfarin as anticoagulation therapy, at a dose of $3.5 \mathrm{mg}$ per day. The latest imaging studies in both recipients revealed patent ePTFE conduits, with satisfactory portal flow and normal postoperative liver function (fig. $1 d$ ).

\section{DISCUSSION}

This is so far the first large series that describes the successful use of an artificial jump graft for portal vein reconstruction in LDLT patients with complete PVT that extends beyond the spleno-mesenteric confluence. In the initial experience of LT surgery, complete PVT was considered an absolute contraindication for LT due to the technical complexity for portal inflow reconstruction and significantly higher mortality rates in such patients. With increased experience in LT and better understanding of the pathophysiology of the PVT, many strategies for reconstruction of graft portal inflow evolved. The surgical technique depends upon the grade of the PVT and its extent, as well as on the type of liver allograft used, e.g. partial or whole. Yerdel et al (7) classified PVT into four grades according to the extent of thrombosis and the severity of luminal occlusion by the thrombus. In patients with PVT grade I or II, the standard surgical strategy is to perform the thrombectomy which may require removal of the innermost layer of the vessel (thromboendvenectomy). In case the affected segment of the portal vein is relatively short, resection of the thrombus bearing segment and primary anastomosis can be successfully achieved

In patients with grade III portal vein occlusion, the thrombosis significantly extends beyond the confluence of the splenic vein and SMV, which poses considerable surgical challenges for portal inflow reconstruction, especially in LDLT. In such patients, portal venous thrombectomy cannot be performed efficiently. Hence, a venous jump graft originating from the patent portion of the SMV or other sources, such as variceal left gastric vein (coronary vein) or splenic vein is a primary alternative. The use of a vascular conduit from the inferior mesenteric vein (IMV) for portal inflow has been reported. However, vascular grafts from other tributaries, such as the coronary vein, has a high risk of anastomotic disruption due to the fragility of the dilated coronary vein, while a vascular conduit from the IMV increases surgical complexity even further. Hence, using a graft vein originating from the patent portion of the SMV remains the preferred approach $(2,3)$.

The options for the vascular graft include the use of either cryopreserved grafts or ePTFE vascular grafts. In the absence of cryopreserved vascular grafts or donor veins, ePTFE synthetic grafts remain a feasible and safe alternative for vascular reconstruction in LDLT $(8,9)$. The indications for synthetic vascular grafts for the splanchnic venous system have been limited because the blood flow in this system is relatively slow and theoretically has a higher propensity to develop luminal thrombosis. However, the haematological condition of the LT recipient is less thrombogenic, due to the impairment of the coagulation parameters. We do not have cryopreserved vascular grafts at our centre, and due to the underlying surgical complexity of harvesting donor or recipient autologous veins during LDLT, we prefer ePTFE vascular conduits for venous reconstruction. In our earlier studies, we have reported the safety and feasibility of ePTFE synthetic grafts for venous reconstruction for vessels such as inferior vena cava and middle hepatic venous tributaries of the right liver allograft without any thrombotic complications (10-12). By far, the role of the ePTFE graft for portal vein reconstruction remains scarce (3). The advantages of the ePTFE synthetic graft are: easy availability and costeffectiveness, differing lengths and diameters can be used in most situations and long-term patency rates of ePTFE grafts are equivalent to cryopreserved vascular conduits. All the patients in this case series had grade III PVT extending to the SMV and requiring portal vein 
reconstruction using the ePTFE vascular graft. In all of the patients, SMV was exposed in the inframesocolic compartment and sufficient length was mobilized and isolated circumferentially. After an end-to-end anastomosis between the ePTFE graft and SMV was performed, the conduit was placed anterior to the pancreas. In the first patient, the anticoagulation consisted only of Aspirin 75 mg once a day. 14 months postoperatively, the ePTFE graft was thrombosed with multiple collateral formation. However, there was no deterioration in the liver allograft function. We changed our protocol for the following two patients that required portal vein reconstruction and both these patients received warfarin therapy with target INR between 2 and 2.5 along with Aspirin 75 mg once daily. At the latest follow up, the portal veins remain patent in two patients, with adequate portal inflow. None of the patients had postoperative ascites formation or gastrointestinal haemorrhage in the postoperative period. The infection of the ePTFE graft is uncommon. However, thrombotic occlusion of the vascular graft and eventual encapsulation of the graft may predispose to septic complications. The first patient in the series had sepsis, which was treated with broad spectrum intravenous antibiotics.

\section{CONCLUSION}

In conclusion, the ePTFE graft remains a feasible alternative as vascular conduit for portal vein reconstruction in LDLT recipients with grade III PVT. Due to the low flow in the splanchnic venous system, anticoagulation is advised for the first two years after LDLT in order to prevent thrombotic occlusion of the graft.

\section{Conflicts of interest}

No conflicts of interest.

\section{Financial source}

No financial Funding from any source.

\section{REFERENCES}

1. Englesbe MJ, Kubus J, Muhammad W, Sonnenday CJ, Welling T, Punch JD, et al. Portal vein thrombosis and survival in patients with cirrhosis. Liver Transpl. 2010;16(1):83-90. doi: 10.1002/t.21941.

2. Francoz C, Valla D, Durand F. Portal vein thrombosis, cirrhosis, and liver transplantation. J Hepatol. 2012;57(1):203-12. doi: 10.1016/ j.jhep.2011.12.034. Epub 2012 Mar 21.

3. Shaw BW Jr, Iwatsuki S, Bron K, Starzl TE. Portal vein grafts in hepatic transplantation. Surg Gynecol Obstet. 1985;161(1):66-8.

4. Hwang HP, Yang JD, Bae SI, Hwang SE, Cho BH, Yu HC. Usefulness of artificial jump graft to portal vein thrombosis in deceased donor liver transplantation. Yonsei Med J. 2015;56(2):586-90. doi: 10.3349/ ymj.2015.56.2.586.

5. Jeng LB, Thorat A, Hsieh YW, Yang HR, Yeh CC, Chen TH, et al. Experience of using everolimus in the early stage of living donor liver transplantation. Transplant Proc. 2014;46(3):744-8. doi: 10.1016/..transproceed.2013.11.068.

6. Jeng LB, Thorat A, Li PC, Li ML, Yang HR, Yeh CC, et al. "V-Plasty" technique using dual synthetic vascular grafts to reconstruct outflow channel in living donor liver transplantation. Surgery. 2015; 158(5):1272-82. doi: 10.1016/.surg.2015.03.018. Epub 2015 Apr 25.

7. Yerdel MA, Gunson B, Mirza D, Karayalçin K, Olliff S, Buckels J, et al. Portal vein thrombosis in adults undergoing liver transplantation: risk factors, screening, management, and outcome. Transplantation. 2000;69(9):1873-81.

8. Hwang S, Ha TY, Ahn CS, Moon DB, Kim KH, Song GW, et al. Reconstruction of inferior right hepatic veins in living donor liver transplantation using right liver grafts. Liver Transpl. 2012;18(2): 238-47. doi: 10.1002/t.22465.

9. Hwang S, Jung DH, Ha TY, Ahn CS, Moon DB, Kim KH, et al. Usability of ringed polytetrafluoroethylene grafts for middle hepatic vein reconstruction during living donor liver transplantation. Liver Transpl. 2012;18(8):955-65. doi: 10.1002/t.23456.

10. Hsu CS, Jeng LB, Thorat A, Yang HR, Yeh CC, Chen TY, et al. Management of extensive retrohepatic vena cava defect in recipients of living donor liver transplantation. Transplant Proc. 2014;46(3): 699704. doi: 10.1016/j.transproceed.2013.11.118.

11. Jeng LB, Thorat A, Yang HR, Li PC. Venous outflow reconstruction in living donor liver transplantation: Dealing with venous anomalies. World J Transplant. 2015;5(4):145-53. doi: 10.5500/wjt.v5.i4.145.

12. Thorat A, Jeng LB, Yang HR, Li PC, Li ML, Yeh CC, et al. Outflow reconstruction for right liver allograft with multiple hepatic veins: "V-plasty" of hepatic veins to form a common outflow channel versus 2 or more hepatic vein-to-inferior vena cava anastomoses in limited retrohepatic space. Liver Transpl. 2016;22(2):192-200. doi: 10.1002/t.24342. 